\title{
Breitband-Biosensor AquaBioTox zur onlinefähigen Trinkwasserüberwachung
}

Thomas Bernard, Thomas Müller, Andreas Jacubasch, Tobias Schuchert

Fraunhofer-Institut für Optronik, Systemtechnik und Bildauswertung IOSB, Karlsruhe

Anke Burger-Kentischer, Tanja Maucher, Georg Geiger, Iris Trick

Fraunhofer-Institut für Grenzenflächen- und Bioverfahrenstechnik IGB, Stuttgart

Fereshte Sedehizade, Berliner Wasserbetriebe, Berlin

\begin{abstract}
Wassernetze sind einer Gefährdung durch absichtliche oder unabsichtliche Verunreinigungen ausgesetzt. Im Rahmen des BMBF-Projektes AquaBioTox wurde ein generisches onlinefähiger Breitband-Toxizitätssensor entwickelt und prototypisch realisiert. Es basiert auf dem „VorkosterPrinzip“: Die Vitalität sehr schnell reagierender, biologischer Kleinstlebewesen, die in einem Bypass der Trinkwasserleitung ausgesetzt werden, wird durch eine Kamera mit automatischer Bildauswertung online überwacht, um signifikante Veränderungen innerhalb kurzer Zeit zu diagnostizieren. Das Monitoringsystem wurde in einer stillgelegten Leitungsstrecke auf dem Betriebsgelände der Berliner Wasserbetriebe getestet. Anhand von repräsentativen Teststoffen wurde die Funktionalität des Breitbandsensorsystems nachgewiesen.
\end{abstract}

\section{Motivation}

Wassernetze sind einer Gefährdung durch absichtliche oder unabsichtliche Verunreinigungen ausgesetzt. Insbesondere in der Trinkwasserversorgung, die zudem ein potenzielles Terror-Angriffsziel darstellt, müssen Gefahren für die öffentliche Gesundheit rechtzeitig erkannt werden. Die Trinkwasserverordnung verlangt deshalb routinemäßige Untersuchungen auf bestimmte Krankheitserreger und chemische Stoffe. Die hierbei eingesetzten Analyseverfahren, die in speziellen Labors offline durchgeführt werden, sind einerseits zu langwierig, um in Fällen von Kontaminationen die Bevölkerung rechtzeitig warnen zu können und wirksame Abhilfemaßnahmen (z. B. Abtrennung betroffener Teile des Wasserversorgungsnetzes) einleiten zu können. Andererseits beschränken sie sich auf ein begrenztes Spektrum. Unbekannte oder nicht erwartete toxische Stoffe, die die Gesundheit des Menschen in gleicher Weise bedrohen können, bleiben unberücksichtigt. Für die frühzeitige Erkennung von gesundheitsbedrohenden Kontaminationen im Trinkwasser wird jedoch, gerade angesichts einer veränderten Bedrohungslage ein onlinefähiges breitbandiges Testverfahren für die Trinkwasserkonformität benötigt, das sehr schnell und zuverlässig reagiert, robust gegenüber Fehlalarmen ist, von Personen ohne wissenschaftliche Qualifikation bedient werden kann und dessen Anschaffungs- und Instandhaltungskosten wirtschaftlich vertretbar sind. Gegenwärtig stehen solche onlinefähigen und breitbandigen Testverfahren bzw. Sensorsysteme nicht zur Verfügung.

\section{Systemkonzept}

Ziel des BMBF-Verbundvorhabens AquaBioTox [1 - 3] war es daher, ein generisches Trinkwasserüberwachungskonzept basierend auf einem onlinefähigen Breitband-Toxizitätssensor zu entwickeln und prototypisch zu realisieren. Das in Bild 1 schematisch dargestellte Biosensorprinzip beruht darauf, aus dem Hauptwasserstrom eine kleine Menge abzuzweigen und damit Indikator-Organismen zu umspülen, deren Vitalität von einer Kamera kontinuierlich beobachtet und automatisch ausgewertet wird.

Um eine hohe Breitbandigkeit hinsichtlich möglicher Giftszenarien zu erreichen und eine hohe Ansprechempfindlichkeit bei geringer Fehlalarmwahrscheinlichkeit zu erzielen, werden mehrere unterschiedliche Biosensorkomponenten in das AquaBioTox-Systemkonzept einbezogen. Dies sind z.B. verschiedene Bakterienstämmen und mammalische Zellen, die bei toxischer Schädigung ihre Fluoreszenz verändern. Da sich die Vitalität der verwendeten Mikroorganismen nicht ausschließlich in 
Abhängigkeit von der Toxizität des Wassers verändert, sondern möglicherweise auch bei anderen unbedenklichen Schwankungen des Wasserzustandes (z. B. Temperatur, $\mathrm{pH}, \mathrm{O}_{2}$-Gehalt etc.), ist es notwendig, die Umgebungsbedingungen der Mikroorganismen automatisiert so zu stabilisieren, dass eine eindeutige Abhängigkeit zwischen anomalen Vitalitätsverhalten der Mikroorganismen und der Toxizität des Wassers aufrecht erhalten wird. Die Online-Diagnose relevanter Vitalitätsmerkmale der Organismen (z. B. Bewegungsgeschwindigkeit, Fluoreszenzverhalten) erfolgt primär mit Hilfe eines bildgebenden Sensors und unter Verwendung modell- und wissensbasierter Methoden der automatischen Bildauswertung. Für die Fusion der verschiedenen heterogenen Sensorinformationen und -daten zu einem belastbaren Gesamtdiagnoseergebnis werden maschinelle Lernverfahren (z.B. basierend auf Kohonen-Karten) angewendet.

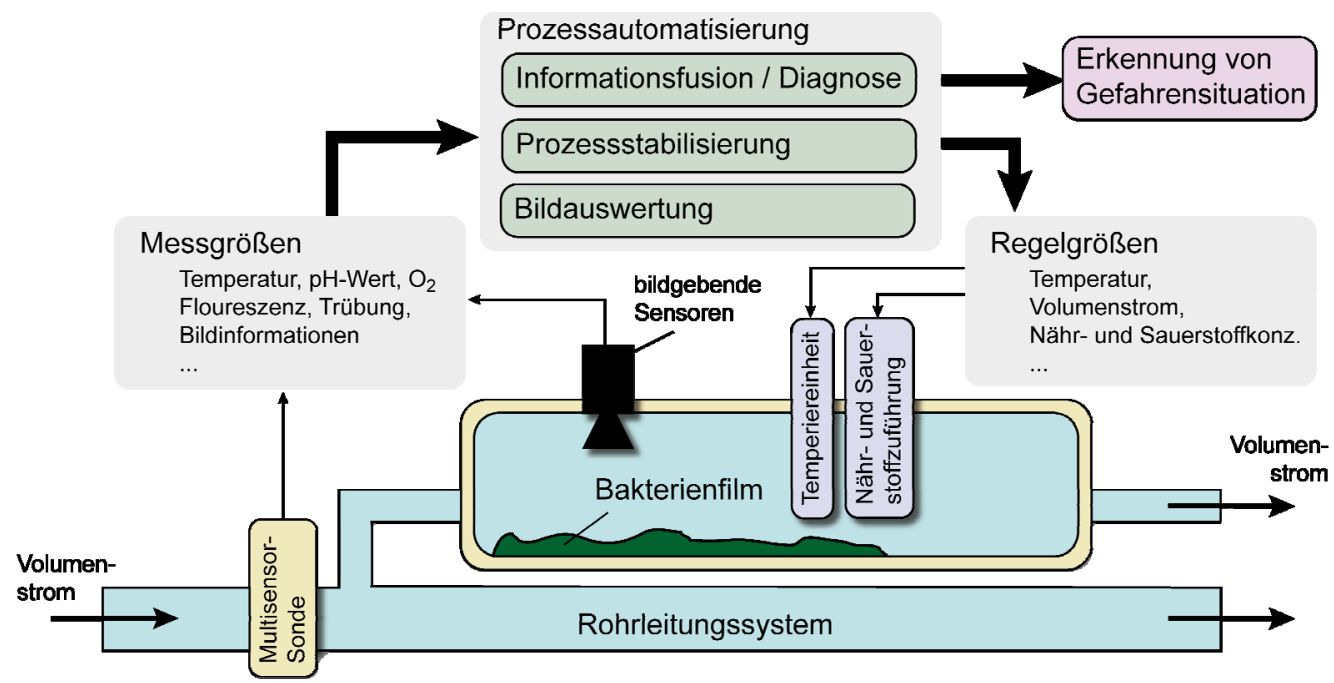

Bild 1: Grundprinzip des AquaBioTox-Sensorsystems zur online-Messung toxischer Inhaltsstoffe im Wasser

\section{Biologische Sensoren}

Die biologischen Sensoren sind das Kernstück von AquaBioTox. Diese reagieren sensitiv auf den Eintrag von Verunreinigungen oder toxischen Stoffen. Als biologische Systeme werden sowohl zwei mikrobiologische Sensoren und zwei mammalische Zellsysteme des Fraunhofer IGB verwendet. Die spezifischen Eigenschaften der biologischen Zellsysteme werden im folgenden näher erläutert (siehe auch $[4-6])$.

Die im Fraunhofer IGB für AquaBioTox etablierten biologischen Systeme sind:

- Escherichia coli RFP-IGB (Bild 1a)

- Caulobacter crescentus RFP-IGB (Bild 1b)

- Hamsterzelllinie CHO-K1-RFP-IGB und humane Zelllinie HEK 293T-RFP-IGB (Fehler! Verweisquelle konnte nicht gefunden werden.)
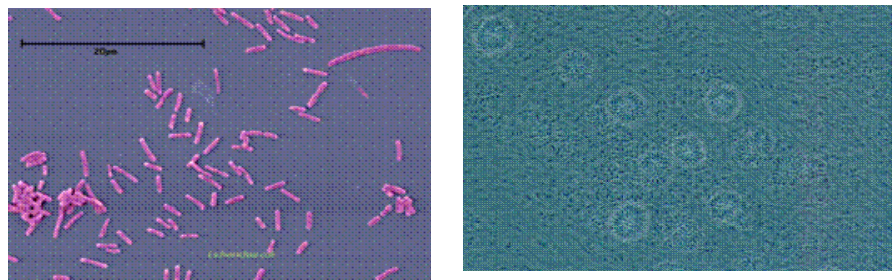

Bild 1: Bakterielle Systeme in AquaBioTox: (a) Zellen von Escherichia coli und (b) Caulobacter crescentus

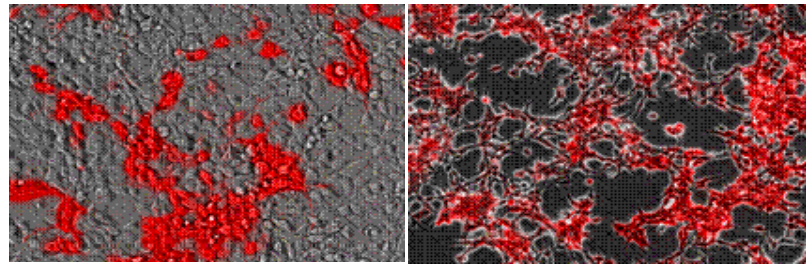

Bild 2: Mammalische System in AquaBioTox: (a) Hamsterzelllinie CHO und (b) humane Zelllinie HEK $293 \mathrm{~T}$. 
Auswahl und Etablierung geeigneter Zellsysteme: Die mikrobiellen Populationen bestehen aus einer Vielzahl mikroskopisch kleiner Zellen, die in hoher Populationsdichte auf kleinstem Raum bereit gestellt werden können und aufgrund der großen Oberfläche einen raschen Stoffaustausch ermöglichen und damit eine sehr rasche Reaktion auslösen können. Die bislang experimentell getesteten Reaktionszeiten liegen bei $<5 \mathrm{~min}$, was für einen späteren Einsatz als Alarmsystem von großer Bedeutung ist.

Zwei Bakterienstämme wurden so modifiziert, dass sie bei Kontakt mit verschiedenen chemischen Substanzen mit einer signifikanten Farb- bzw. Fluoreszenzänderung reagieren, die mit einer Fluoreszenzmesssonde aufgenommen und ausgewertet wird. Um die Breitbandigkeit zu erhöhen, wurden zwei mammalische Zelllinien mit demselben Fluoreszenzmarker versehen. Damit wird eine größere genetische und metabolische Nähe zu menschlichen Organismen erreicht und das Reaktionsspektrum abgesichert und erweitert.

Testsysteme: Die biologischen Sensoren werden im immobilisierten Zustand auf verschiedenen Trägermaterialien in Bioreaktoren (20 ml-Volumen) realisiert, in denen die Messungen mit der Fluoreszenzsonde aufgenommen werden. Bild 4 zeigt die makroskopisch wahrnehmbare Farbänderung hier bei dem Testorganismus Escherichia coli RFP-IGB, zu beobachten auf zwei verschiedenen Trägermaterialien.
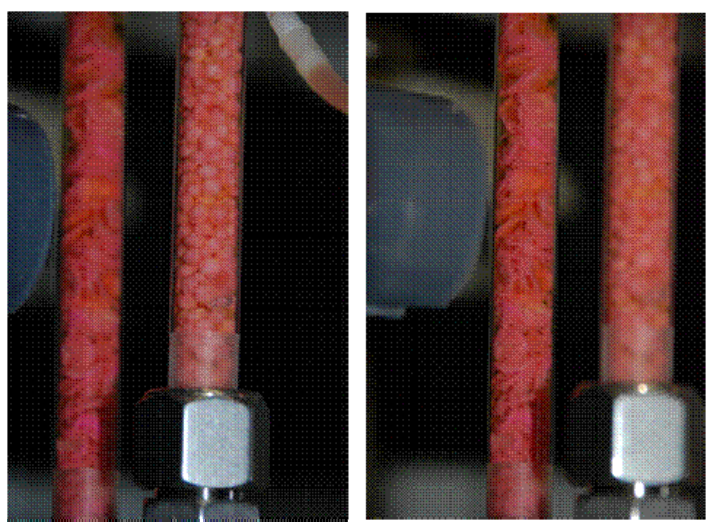

Bild 4: Messzelle mit den immobilisierten Mikroorganismen: Fluoreszenzänderungen mit bbe-Sonde messbar. Makroskopisch sichtbare Farbänderungen von pink (links) nach orangefarben (rechts) nach Zugabe von Testsubstanz.

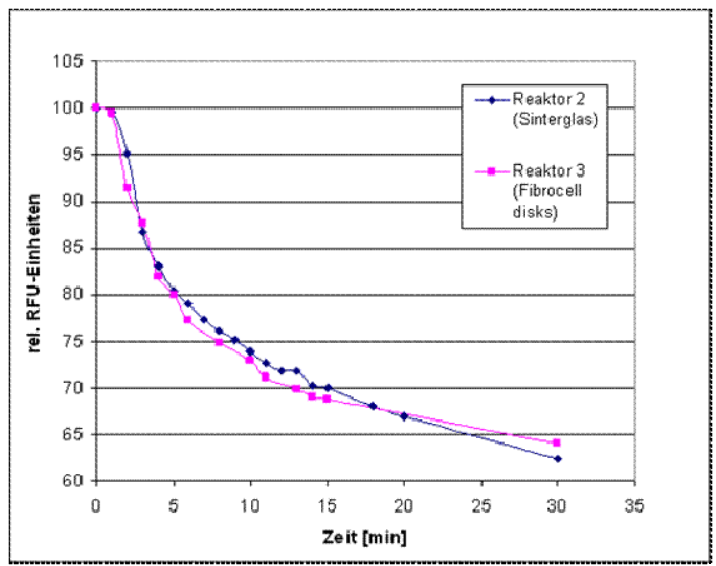

Bild 3: Prüfung der Funktionalität des Testsystems und der Sensitivität der Testorganismen: Zwei verschiedene Trägermaterialien zur Immobilisierung.

Fluoreszenzabnahme nach 3 - 5 min deutlich erkennbar. (Testorganismus Escherichia coli RFP-IGB).

Prüfung auf Sensitivität: Die Sensitivität wurde für eine Reihe relevanter Substanzen geprüft. Die Messwerte wurden dabei mit einer für den Wellenlängenbereich geeigneten Fluoreszenzsonde der Fa. bbe Moldaenke aufgenommen. Aus Fehler! Verweisquelle konnte nicht gefunden werden. ist zu entnehmen, dass bei Zugabe einer Testsubstanz die Signaländerung bereits nach 3 - 5 Minuten deutlich erkennbar ist.

Stabilität der Zellsysteme: Die Stabilität für die mikrobiellen und mammalischen Zellsysteme kann mindestens für 3 Wochen (maximal getesteter Zeitraum für die verwendeten Bakterienkulturen) aufrecht erhalten werden. Mit den mammalischen Zelllinien wurden Vitalität und Fluoreszenz auch nach 10-wöchiger Inkubation nachgewiesen. 


\section{Automatisierung, Bildauswertung und Sensorfusion}

Um optimale Vitalitätsbedingungen für die Mikroorganismen sicherzustellen und somit eine nachhaltige Nutzung des Sensorsystems zu gewährleisten, ist eine Stabilisierung der natürlichen Schwankungen des Wasserzustandes notwendig (insbesondere Volumenstrom, Temperatur, Zuflussrate der Substratlösung). Hierzu wurden entsprechende Regelungskonzepte realisiert.

Die am Fraunhofer IITB entwickelten Komponenten zur automatischen Bildauswertung umfassen:

- die rechnergestützte Überwachung der Biosensorkomponenten mit bildgebender Sensorik,

- die Modellierung des Zusammenhangs von Messungen (Beobachtungen) und Systemzustand (Lebendigkeit der Indikatororganismen, Wassergüte),

- die automatische Erfassung der vitalitätsanzeigenden Parameter,

- die Generierung eines Ausgangssignals über den Sicherheitszustand.

Die Herausforderung bestand darin, auch schwache Vitalitätsveränderungen der Indikatororganismen (etwa bei langsam wirkenden Toxinen) robust erkennen und sie von Störeinflüssen und natürlichen Schwankungen des Trinkwassers zuverlässig unterscheiden zu können.

Für die Daphnien-Biosensorkomponente wurde eine robuste Bildauswertungskomponente entwickelt, welche die Daphnien von sich mitunter im durchgeleiteten Wasserstrom bewegenden Luftblasen und Exuvien sowie von sonstigen Hintergrundartefakten unterscheiden kann. Bild 6 zeigt hierzu zwei Beispiele, bei denen in den Wasserkammern zum Test des Algorithmus absichtlich viele Störeffekte erzeugt wurden. Die berechneten Farben decken sich mit den realen Gegebenheiten. So wird insbesondere auch die sich bewegende große Luftblase im linken Bild (oben rechts in der rechten Kammer) korrekt erkannt.
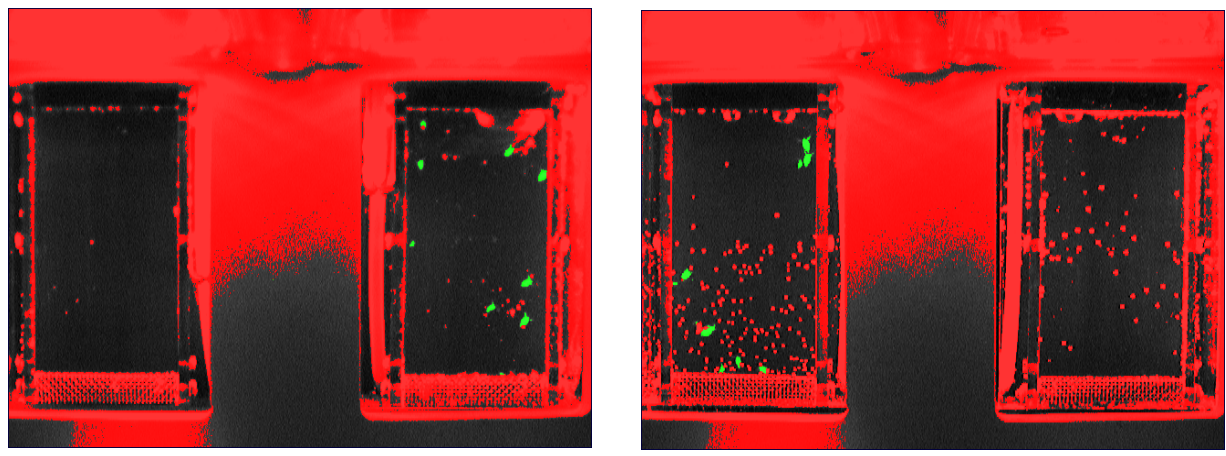

Bild 6: Beispiele zur Unterscheidung der Daphnien von sich z.T. im Wasserstrom bewegenden Luftblasen, Exuvien und Artefakten. Grün: algorithmisch detektierte Daphnien. Rot: algorithmisch ermittelte Vordergrundstrukturen, die keine Daphnien sind.

Für die Bakterien- und Zellsystem-Biosensorkomponente wurden Verfahren zur robusten Auswertung der räumlichen und zeitlichen Fluoreszenzverteilung der Kulturen entwickelt.

Um einerseits hohe Sensitivität und schnelles Ansprechen bei bedrohlichen toxischen Veränderungen des Trinkwassers zu gewährleisten und andererseits ausreichende Robustheit gegenüber kostenaufwändigen Fehlalarmen sicherzustellen, ist es notwendig, die Bildinformationen des biologischen Breitbandsensors mit den Daten von weiteren relevanten Sensoren (z.B. Temperatur, pHWert, Sauerstoffgehalt, Leitfähigkeit) intelligent zu fusionieren. Für die Fusion der heterogenen Sensorinformationen $\mathrm{zu}$ einem belastbaren Gesamtdiagnoseergebnis wurden modell- und datengetriebene Methoden (z.B. Neuronale Netze, Selbst-Organisierende Karten) verwendet [7]. Die relevanten Sensormerkmale werden so fusioniert, dass die statistische Signifikanz der jeweiligen Sensormerkmale berücksichtigt wird. Dadurch wird eine robuste Erkennung von Gefahrensituationen gewährleistet. 


\section{Demonstrator und Teststrecke}

Zur Untersuchung des Demonstrators auf seine Funktionsfähigkeit unter praktischen Bedingungen wurde eine Versuchsanlage auf einer stillgelegten Leitungsstrecke aufgebaut (Bild 7). Alle Merkmale der Leitungsstrecke (Material, Alter, Nutzung und Fließverhältnisse wie Druck und Durchfluss) entsprechen einer üblichen Trinkwasserleitung und sind somit repräsentativ für Trinkwasserversorgungsnetze in Berlin und in Deutschland. Die Versuchsanlage erfüllt alle Bedingungen und Variationsmöglichkeiten für die Durchführung von Testabläufen. An der Versuchsanlage wurden nicht nur die Funktionsfähigkeit, die Zuverlässigkeit und Robustheit des Demonstrators (und seine Einzelkomponenten) getestet sondern auch die Bedienung, die Anfälligkeit und der Wartungsaufwand unter Praxisbedingungen.
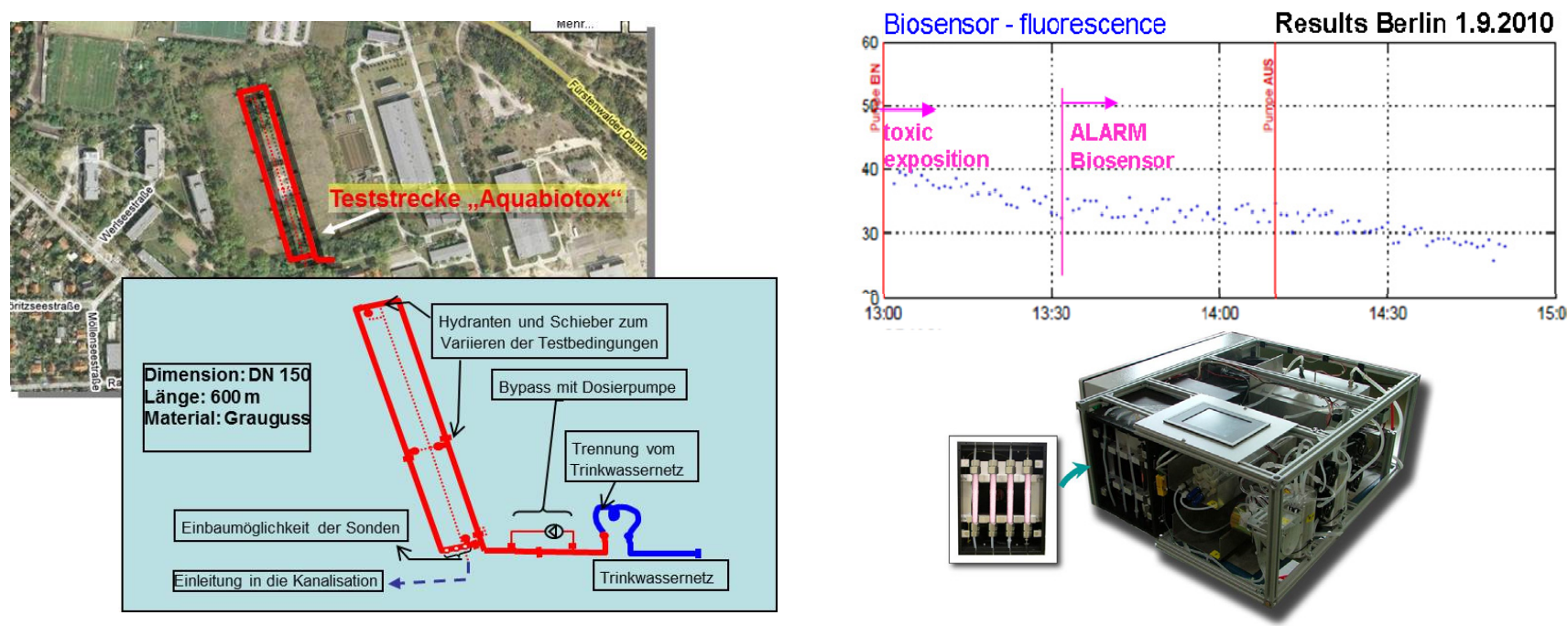

Bild 7: Demonstrator auf dem Gelände der Berliner Wasserbetriebe in Berlin-Friedrichhagen (links) und Ergebnisse eines Tests (rechts oben). Ein Prototyp des AquaBioTox-Sensors ist unten rechts gezeigt

\section{Literatur}

[1] BMBF-Projekt AquaBioTox: Onlinefähige Trinkwasserüberwachung auf der Grundlage eines biologischen Breitbandsensors mit automatischer Bildauswertung. Laufzeit: 1.12.2007 - 31.11.2010

[2] I. Trick: Überwachung von Trinkwasserleitungen. Jahresbericht des Fraunhofer-Instituts für Grenzflächenund Bioverfahrenstechnik, Stuttgart 2009/2010, 86-87

[3] Fereshte Sedehizade, Iris Trick, Anke Burger-Kentischer, Tanja Maucher, Georg Geiger, Thomas Bernard, Helge-Björn Kuntze, Thomas Müller, Felix Sawo, Christian Moldaenke: On-line Monitoring of of Drinking Water Based on a Biological Broad-Spectrum Sensor with Automatic Image Evaluation (AquaBioTox). In: Elsner, Peter (Ed.); Fraunhofer Allicance for Defense and Security Research VVS: Future Security : 4th Security Research Conference Karlsruhe : September 29th - October 1st, 2009, Karlsruhe : Fraunhofer IRB Verlag, 2009, pp. 363-374

[3] T. Maucher, I.Trick: Biologische Toxine in Wasser - Nachweis mit Biosensoren. Jahresbericht des Fraunhofer-Instituts für Grenzflächen- und Bioverfahrenstechnik IGB, Stuttgart 2010/2011, 106-107

[4] T. Maucher, G. Geiger, A. Burger-Kentischer, I. Trick: Influence of Substances of different origin on fluorescence of a whole cell sensor. European Summerschool "Proteomics Basics", Brixen (I), August 2011

[5] T. Maucher, G. Geiger, A. Burger-Kentischer, I. Trick: Detection of B- and C-Substances in the Water Supply System using a novel Biosensor System Dechema: European Congress of Applied Biotechnology, Berlin, September 2011

[6] F. Sawo: Lernfähiges Monitoringsystem zur Detektion toxischer Stoffe in Trinkwassernetzen. Jahresbericht Fraunhofer IOSB 2009/2010, S. 64 - 65 